\title{
L'ereignis, Le Mot-Directeur De La Pensée De Heidegger
}

\section{Pascal Dieudonné Roy-Ema}

De La Enseignant-Chercheur-

Doctorat en Philosophie de IESASTRES.a ecolPoitiers, France

Université Alassane Ouattara, Bouaké, Côte d'Ivoire

doi: 10.19044/esj.2017.v13n23p234 URL:http://dx.doi.org/10.19044/esj.2017.v13n23p234

\begin{abstract}
For over sixty years (from 1910 to 1973), Martin Heidegger carried out a work of thought which led him to create a large quantity of neologisms. It also led him to create a new use of idioms in the German language. This was regarded as a renewed vocabulary. His study bears new meanings and expresses the philosopher's work of thought and the new concepts he proposes. Among them is the Ereignis that this text proposes to question the content. The Ereignis is what makes time and being belongs to each other. It is the relationship of all the relations engendered by this co-membership. This is made possible by the difference installed at the heart of the same. Heidegger himself admitted that the Ereignis was the keyword of his whole thought since the early 1930s. It is the word-director of his thought.
\end{abstract}

Keywords: Ownership, Dasein, Ereignis, Being, Native time

\section{Résumé}

Martin Heidegger a réalisé pendant une soixantaine d'années (de 1910 à 1973) un travail de pensée qui l'a conduit à créer une grande quantité de néologismes, ainsi qu'un nouvel usage d'idiomes de la langue allemande, un vocabulaire renouvelé, porteur de sens nouveaux, exprimant le travail de pensée du philosophe et les nouveaux concepts qu'il propose, parmi lesquels ici, l'Ereignis que ce texte se propose de questionner le contenu. L'Ereignis est ce qui fait s'entr'appartenir le temps et l'être. Il est le rapport de tous les rapports engendrés par cette co-appartenance. Celle-ci est rendue possible par la Différence installée au cœur du Même. De l'aveu même de Heidegger, l'Ereignis est le mot-clé de toute sa pensée depuis le début des années 1930; c'est le mot-directeur de sa pensée.

Mots-clés: Appropriation, Dasein, Ereignis, Être, Temps originaire 


\section{Introduction}

Martin Heidegger, philosophe allemand du XXè siècle, a réalisé pendant une soixantaine d'années (de 1910 à 1973) un travail de pensée qui l'a conduit à créer une grande quantité de néologismes, ainsi qu'un nouvel usage lexical de la langue allemande, un vocabulaire renouvelé, porteur de sens nouveaux, exprimant le travail de pensée du philosophe et des nouveaux concepts qu'il propose, parmi lesquels ici, l'Ereignis. La traduction de l'œuvre de Martin Heidegger en France a conduit à innover dans le domaine de la langue philosophique, non sans difficultés, mais en permettant, par ce travail de traduction/interprétation, une réflexion approfondie sur la pensée du philosophe.

Pour Martin Heidegger, la métaphysique occidentale tout entière est marquée par une confusion complète entre l'être et l'étant, par une incapacité de penser l'être. Pourtant, une importante tradition a toujours dénié à l'ontologie un rôle de premier plan. L'analyse des textes de Martin Heidegger (en particulier les Beiträge zur Philosophie, jamais traduits en français) consacrés à cette tradition platonicienne montre qu'il n'a jamais pris au sérieux l'effort néoplatonicien. La fin du volume est consacrée à une thèse en vogue dans le monde heideggérien; certains auteurs se plaisent à souligner une certaine identité entre le concept d'Ereignis ("advenir") et celui de l'Un chez Plotin. Une discussion serrée montre qu'il n'en est absolument rien (Narbonne Jean-Marc, 2001). Que révèle-t-il donc exactement, ce concept d'Ereignis chez Martin Heidegger ? N'est-il pas le mot-directeur de la pensée heideggérienne? Telle sera l'orientation fondamentale de cet article.

$\mathrm{Au}$ cours de cet exercice, nous nous appliquerons, essentiellement, à dégager le sens de la notion d'Ereignis chez Martin Heidegger, carrefour fulgurant de la pensée métaphysique moderne, en tirant la conséquence de ce que l'Ereignis est l'élément clé, fondateur et unificateur de sa pensée.

\section{Décryptage du concept d'Ereignis}

Le mot «Appropriation» essaie de traduire l'allemand Ereignen, l'un des termes fondamentaux mais difficilement traduisibles du lexique heideggérien, d'où découle le concept d'Ereignis. " Appropriation» signifie d'abord prendre appui sur, das Eigene entendu « comme mouvement d'amener une chose à son propre ». Nous ne sommes pas dans le registre notarial de la « propriété », mais plutôt dans celui de l'expression française « remettre en main propre $»$.

À travers le terme « approprier », il ne faut pas entendre qu'une chose devienne la propriété ou la possession, mais le fait d'« amener quelque chose à être ce qu'elle est » (Heidegger, 1988, p.28) ou la mener à son terme. " Appropriation » qualifie aussi le « demeurer ensemble ». À propos de 
l'exemple de l'être de la cruche tiré de la conférence sur la Chose, Didier Franck conclut : « le ciel et la terre, les divins et les mortels sont appropriés ou s'approprient réciproquement dans la mesure où aucun d'entre eux ne va sans les autres, dans la mesure où, pour parler de manière grecque, chacun reçoit son « être » de celui des autres auxquels il est ainsi (confié) » (Franck, 2004, p. 75).

Plus profondément, «Appropriation » qualifie l'Ereignis en ce qu'il conduit l'homme à ce qui lui est propre (Ereignen) en « le plaçant dans un rapport - celui de la pensée comme essence de l'homme, et de l'être de l'étant - que l'on peut proprement qualifier d'abyssal, dans la mesure où aucun de ses termes ne lui préexiste, mais où chacun se définit comme relation à l'autre » (écrit J. Pieron, 2010, p. 128).

Ereignis est le mot, allemand, posé par le penseur Martin Heidegger, pour désigner la foncière co-appartenance entre "être " et 《temps ». Ereignis n'est pas une entité en soi, ce serait davantage un libre-jeu pluridimensionnel dans lequel être et temps seraient livrés l'un à l'autre, en accédant ainsi l'un par l'autre à leur caractère propre, dans un mouvement abolissant toutefois l'un et l'autre au profit de leur indissociable unité; indissociable unité ouvrant alors sur l'aube d'une nouvelle appréhension de « être » et « temps » en tant que libre-jeu des Quatre.

Le Quatre ou Quadriparti est le jeu de renvoi, jeu de miroir incessant par lequel toute chose est ; à savoir : les mortels, les signes en provenance du divin (les indices du sacré), le ciel et la terre. Ereignis est, en fait, une sorte d'origine abyssale à ce don qu'est l'être. Ereignis est ce qui donne temps, dans l'entr'appartenance foncière entre « temps » et « être ». « Temps » étant ce qui donne « être », dans l'entr'appartenance foncière entre « être » et « temps ». Cette double donation a pour source Ereignis. Ereignis en tant que, non pas une entité constituée, mais en tant que : libre-jeu jouant dans l'Ouvert, d'une donation provenant de l' « être » et issue du « temps », dans la relation à une provenance encore plus originaire, la donation elle-même (Ereignis).

Tout ceci en intégrant le fait que, du point de vue de l'histoire de l'« être »dans le déploiement de la pensée occidentale depuis son origine grecque, ce qui caractérise fondamentalement l'« être » en tant que donation, c'est que l'« être » se voile, se garde en retrait, dans le mouvement même où il se donne en son déploiement. Comme le dit très bien, Marlène Zarader:

«(...) Et c'est seulement lorsque la pensée ne se contente pas de reconnaître le retrait du Es dans la donation, mais qu'elle s'avance jusqu'à penser ce Es (c'est à dire l'Ereignis) comme un essentiel « dépropriement » de lui-même, que, comme le dit encore Heidegger, « le voilement ne se voile plus ». Il ne se voile plus, c'est-à-dire qu'il 
apparaît enfin comme ce qu'il est : voilement irréductible, retrait essentiel. » (1986, p. 249).

En en arrivant là, on ne peut résister à faire le rapprochement entre cette perspective de pensée et celle du grand penseur indien Nagarjuna dans la présentation qu'en fait Jean Varenne dans l'Encyclopédia Universalis: « Nagarjuna concède que les phénomènes sont réels, mais seulement en tant que phénomènes ; on a raison de dire qu'ils sont comme un voile, mais à condition de comprendre qu'il n'y a rien derrière le voile. ") (Varenne Jean, 2017).

De l'allemand Das Ereignis, le mot Ereignis signifie au sens courant l'événement, ce qui arrive. Martin Heidegger l'entend comme er-eignis-, ce qui amène à être proprement soi, sa propriété. Ereignen est pris au sens de « faire advenir à soi »(Heidegger, 1976, p. 227). Le mot signifie à la fois laisser advenir à soi, laisser-être et manifester de la bienveillance. Un sens métaphorique approché peut être le père qui protège son enfant, le conseille sans le contraindre, mais en le laissant développer sa propre personnalité, être ce qu'il doit être en toute liberté.

Heidegger désigne aussi l'Ereignis par un « il y a être », Es gibt Sein, c'est-à-dire, comme l'événement d'une pure donation. L'être donne l'étant et se retire au profit du donné. L'Ereignis reste caché derrière le voilement inhérent à « l'être-là » comme « être-au-monde ». " En se décelant dans l'étant, l'être disparaît comme «Ereignis » et apparaît comme être de l'étant. Ce qui se retire n'est donc pas l'être comme être de l'étant, mais l' " Ereignis ", comme événement de la Lichtung des Seins » (Piéron, 2010, p. 128-129).

Ereignis est un terme auquel Heidegger a accordé, à partir des années 40, beaucoup d'importance. Littéralement, il signifie événement. C'est ainsi qu'il est parfois traduit. Le verbe correspondant, Ereignen, signifie effectivement " se produire », " survenir ». Mais le verbe Eignen, qui se trouve dans Er-eignen, signifie également adapter et avec Sich eignen, « convenir pour ceci ou cela ».

Heidegger a voulu qu'on entende Ereignis à la fois comme événement et comme «convenance». À l'origine, ces mots proviennent d'un terme - Eräugen - qui signifie « (se) laisser voir », " (se) manifester ». Quand cela a lieu cela constitue effectivement un événement et le sens de cet événement est qu'il y a convenance entre celui qui voit à la chose qui est vue.

Mais, dans le texte de Heidegger, il ne s'agit pas du voir. C'est l'homme lui-même et l'être qui, d'une certaine manière, conviennent, " correspondent ». 
L'Ereignis est cet événement, ce mouvement en vertu duquel, en s'entre-appartenant l'un l'autre, l'homme et l'être sont portés à la plénitude de leur « propriété », de ce qui leur est propre.

Mais pourquoi l'Ereignis et quelle signification idéologique nous nous sentons contraint de lui attribuer?

En 1949, Heidegger, à Jean Beaufret, a déclaré ceci : dans l'Ereignis « l'homme est transi par l'être jusqu'à en devenir Dasein et cet Ereignis est la vérité de l'être auquel le Dasein appartient essentiellement » (J. Beaufret, 1985, p. 126)

La «vérité de l'être » est ainsi que les hommes sont sourds à la question de l'être. Il y a Ereignis, événement appropriant, quand l'homme, apercevant son « manque à être », son peu d'humanité authentique, devient Dasein et s'ouvre ainsi à la question et à la vérité de l'être.

$\mathrm{Au}$ fond il y a Ereignis quand un homme devient un être acharné et son implication dans son monde devient un moment du mouvement d'appropriation de l'homme et de l'être; c'est participer à la restitution d'une humanité corrompue et détruite par les ruses de l'existence inauthentique.

C'est par avenance que François Fédier rend le terme Ereignis dans sa traduction des « Beiträge zur Philosophie » (vom Ereignis). Fédier traduit ainsi le titre : Apports à la philosophie - De l'avenance. Quelque chose advient selon une événementialité appropriante.

Le génitif, « De l'Ereignis », doit être entendu en son double sens, donc aussi comme « à partir de l'Ereignis », dans la mesure où la pensée est justement pensée par Heidegger comme un envoi appartenant à l'histoire de l'Être, donc venant de l'Ereignis lui-même. C'est pourquoi François Fédier précise :

«Comprendre ainsi le premier mot du titre permet de l'entendre comme Heidegger sans aucun doute l'entend lui-même. Ces Apports à la philosophie, ce ne sont donc pas les «contributions du professeur Heidegger », mais bien - ouvrons à notre tour les oreilles : Ce que l'Ereignis (soi-même) apporte à la philosophie. Il faut donc, me semble-t-il, rendre ainsi le titre complet du livre: Apports à la philosophie/De l'Ereignis ». (F. Fédier, 2013, pp. 142-143).

Nous sommes une fois de plus perplexes et ne voyons guère en quoi le mot d'apport permet de lever le moindre contre-sens possible, soulignait Etienne Pinat (Etienne Pinat, 2014). Si l'on dit de l'Ereignis qu'il apporte quelque chose à la philosophie, on peut tout aussi bien dire que l'Ereignis contribue à la philosophie, et que cet ouvrage en présente les contributions. De la même façon, dire que ce sont les apports à la philosophie n'empêche nullement de penser qu'il s'agisse seulement d'un « apport personnel », comme on dit lorsqu'on emprunte pour acheter. Mais 
cette traduction est plus grave encore, car une fois de plus il s'agit par elle d'imposer une compréhension déterminée du titre, là où le lecteur devrait être laissé libre de comprendre, et de se méprendre. En effet, en allemand, le lecteur qui lit «Beiträge» peut penser qu'il s'agit simplement là des contributions de Heidegger, mais précisément Heidegger ouvre son livre sur un texte visant à corriger cette première compréhension du titre. Ainsi, il écrit:

«L'intitulé public va nécessairement s'entendre, pour l'instant, de manière terne, commune et peu significative, et laisser croire qu'il s'agit ici de «contributions » «scientifiques » au «progrès de la philosophie ».[...] C'est pourquoi le titre approprié s'énonce $D e$ l'Ereignis. Cela ne dit pas que l'on en fait un compte-rendu ni que l'on disserte à ce sujet, mais dit tout au contraire : De l'Ereignis, vient-à-soi une pensante et parlante appartenance à l'Être et à la parole « de » l'Être » (F. Fédier, 2013, pp. 15-16).

Par conséquent, en voulant traduire par « apports » plutôt que par « contributions » pour empêcher de mal comprendre le titre, François Fédier devance Heidegger, lui coupe l'herbe sous le pied et l'empêche de s'expliquer simplement comme il le fait pour le lecteur allemand. Traduire par «contributions», c'est justement proposer une traduction qui corresponde parfaitement à l'allemand, et qui permette au lecteur français de se trouver dans la même situation que le lecteur allemand, comprenant mal le tire à la première lecture et le comprenant mieux ensuite après lecture des explications fournies par Heidegger lui-même.

L'autre élément du titre qui frappe dès la couverture, c'est bien évidemment cette étrange traduction d'Ereignis par «avenance », qui est cependant un progrès dans la mesure où la traduction de 1997 proposait « amêmement », sans doute pour rendre l'idée de « rendre même ». Le mot est généralement traduit par « événement», " événement-appropriant » ou encore «appropriement», ou bien n'est pas traduit du tout, comme pour Dasein. Mais personne, à part une poignée de commentateurs d'une école bien précise, ne traduit Ereignis par «avenance », et même Gérard Guest a dit dès 2005 dans la revue L'Infini son opposition franche à cette interprétation de l'Ereignis, car il s'agit une fois de plus d'une interprétation qu'on cherche à imposer.

Cette traduction a été proposée en 2004 à Lausanne, et la moindre des choses serait qu'elle soit expliquée dans la traduction des Beiträge, ce qui n'est jamais le cas, empêchant le lecteur qui n'a pas la chance de posséder «Entendre Heidegger» de François Fédier dans sa bibliothèque d'entendre quelque chose aux Beiträge. Cette conférence, mettant en scène dramatiquement la trouvaille comme un déclic soudain («Henri Crétella me parlant, il m’est soudain apparu comme par déclic... », (F. Fédier, 2013, p. 
154)) nous apprend que cette traduction, faisant une fois de plus retour à l'ancien français («En français, en bon français depuis le XIème siècle... » (F. Fédier, 2013, p. 155)) lui a été suggérée par Henri Crétella en prenant pour prétexte le fait que Heidegger, dans la «Lettre sur l'humanisme », écrit que l'Être est «l'avenant», et l'écrit en français pour traduire das Ankommende.

Mais comme pour la traduction de Dasein par « être le là », à vouloir être plus heideggérien, que Heidegger, on finit par le trahir, car « l'avenant » n'est justement pas ici une traduction d'Ereignis et nulle part il n'est écrit que cet ankommen doive être purement et simplement identifié à l'Ereignis. Se rendant compte de son audace, François Fédier écrit : « Il m’arrive de penser à la moue qu'aurait faite Heidegger m'entendant lui soumettre cette proposition » (F. Fédier, 2013, p. 156). Nous comprenons pourquoi Heidegger aurait fait la moue et pensons que François Fédier aurait dû y penser plus, car il y a de quoi.

Maintenant, en quoi la traduction par « avenance » permet-elle de rendre en français ces trois nuances ? On n'y trouve ni allusion à l'œil ni allusion au thème du propre. Seule la venue, donc l'événement, se laisse apercevoir. Mais dans ce cas, pourquoi ne pas traduire tout simplement par « événement» et dispenser le lecteur de cette trouvaille archaïsante ? Sa nécessité n'apparait nullement, elle donne plutôt l'impression de la trouvaille d'une érudition pédante dont on pourrait nous épargner afin d'alléger (et non d'allégir) la traduction, renchérit Fédier.

En 2004, François Fédier terminait sa conférence sur la traduction de la formule «das Ereignis ereignet» par «l'avenance appareille». Fort heureusement, elle n'a finalement pas été retenue dans la présente traduction où elle est rendue par « l'avenance fait venir à soi » (F. Fédier, 2013, p. 399). Il faut entendre ici cet accord dont nous parlions plus haut, à savoir que l'Ereignis ereignet, c'est-à-dire approprie l'un à l'autre le Da-sein et l'Être et les fait ainsi venir à soi, à ce qu'ils ont en propre.

Le montrer de la Dite est l'approprier : das Ereignen. Il est le proprier (Eignen) qui apporte (er-bringt) le déploiement de l'être et la dimension de l'absence. L'approprier est ce proprier apportant (das erbringende Eignen). Comme approprier (Er-eignen), il apporte (er-bringt) absence et présence, c'est-à-dire qu'il livre (er-gibt) le libre jeu de l'éclaircie dans lequel ont lieu ces déploiements. Ce que livre l'approprier de telle manière, soit par la Dite (durch die Sage), est ce qu'il y a de plus octroyant (das Gewährende), puisqu'il s'agit du don du libre jeu du monde, où les quatre contrées parvenues à leur propre accordent toutes choses.

Cet approprier est alors 1'événement-appropriement (das Ereignis), dont la générosité réside en ce que, tout en se retirant, il donne être et temps, présence et absence. Il est ce qu'il y a de plus octroyant, il est la donation- 
même (das Er-gebnis), dont le don accorde ce qu'il y a (Es gibt). Il est la donation dont l'être même a besoin pour parvenir à son propre comme présence. En même temps, l'Ereignis lui-même est affecté par les dimensions de son don, de telle sorte qu'il se trouve en fin de compte luimême déterminé par elles : donnant il se soustrait, se retient en lui-même, se retire. Il est toujours et aussi Enteignis (dé-propriement), c'est-à-dire l'anonyme, le ça, le Es du Es gibt.

Cet événement-appropriement, en sa double dimensionnalité et en son double don, est donc le proprier qui remue la Dite dans son montrer.

L'événement-appropriement, aperçu dans le montrer de la Dite, ne se laisse représenter ni comme un événement, ni comme un avoir-lieu, mais se laisse seulement éprouver comme l'octroyant dans le montrer de la Dite (W.J. Richardson, 2011, p. 258).

L'Ereignis se laisse éprouver comme ce qui se remue (sich regt) dans le montrer de la Dite, comme ce qui, se remuant, émeut (er-regt) la Dite (c'est pourquoi la Dite - et le mot - est libérante). Il est ce qui, dans le montrer de la Dite, parvient à son propre, qui est d'être le plus octroyant, qui est d'être celui qui approprie chaque chose. Ainsi, l'Ereignis, parvenant à son propre dans la Dite, y règne. Laissant l'Ereignis se déployer en elle, la Dite libère celui-ci, lui permet de déployer à son tour tout ce qui est en son essence propre, d'accorder le séjour où chaque chose va venir se déployer pleinement. L'Ereignis laisse alors chaque chose, mortels y compris, venir en son propre en présence, à la place qui lui appartient en propre. La Dite, laissant le proprier de l'Ereignis se remuer en elle, est le mode le plus propre de l'approprier.

«La Dite reposant dans l'événement-appropriement est, en tant que Montrer, le mode le plus propre de l'approprier » (W.J. Richardson, 2011, p. 262).

La Dite montrante (die zeigende Sage), qui délivre l'Ereignis de la sorte, est le déploiement de la parole proprement dit. L'Ereignis est parlant (sagend), c'est-à-dire qu'il dit, qu'il montre. La Dite est le mode, la mélodie (die Weise, $\mu \dot{\lambda} \lambda o \varsigma$ ) en lequel parle 1'Ereignis. La parole en son déploiement le plus propre, soit comme Dite, est la mélodie de l'événement-appropriement.

La parole parle. Elle parle comme Dite, comme montrer appropriant. Elle parle comme mode le plus propre de l'Ereignis. La parole parlant de la sorte est seule à véritablement parler, elle parle solitairement. Elle a comme trait le plus originel, ainsi que l'a reconnu le poète Novalis, de ne se soucier que d'elle-même. Elle parle solitairement, c'est-à-dire n'a pour seul souci de permettre aux choses de se déployer en leur essence propre. Ce qui ne la prive pas de tout rapport, au contraire, la parole s'est laissée expérimentée comme le rapport de tout rapport. 
Pourtant « ne peut être solitaire que ce qui n'est pas seul ; pas seul, c'est-à-dire pas séparé, isolé, sans relation » (W.J. Richardson, 2011, p. 265).

De quoi est-il question avec l'Ereignis, s'interroge Etienne Pinat? (Etienne Pinat, 2014)

Il s'agit du mot directeur de la pensée de Heidegger depuis 1936, mot qu'on peut comprendre selon au moins trois points de vue. Ereignis est un mot courant de l'allemand qui veut dire « événement», et l'Ereignis doit bien être entendu ainsi, mais il ne s'agit pas d'un événement qui arrive à un moment précis du temps et de l'histoire, en lieu précis du monde, comme le font les autres événements. Il est l'événement par excellence par lequel sont possibles les événements, et d'événement comme lui, il n'y en a qu'un, car il est l'événement par lequel a lieu le temps, l'histoire comme histoire de l'Être, et le Da-sein lui-même. Il est ce par quoi «il y a» temps, être, histoire, et cet « il y a » signifie «ça donne » (es gibt), l’Ereignis étant cela qui donne. On peut donc traduire Ereignis par « événement », c'est le choix fait dans les traductions américaines, italiennes et espagnoles, ce choix ayant le mérite d'être le décalque du terme allemand, qui sera ensuite compris plus précisément lors de la lecture de l'ouvrage, exactement comme le fait le lecteur allemand. On peut aussi, comme le fait Gérard Guest (2005, pp. 306372), lui adjoindre une majuscule, pour faire signe vers le caractère singulier de cet événement.

Mais l'Ereignis signifie aussi en allemand un Er-eignis, dans lequel il faut entendre eigen, le propre, donc une manière de rendre propre, l'événement d'un appropriement qui fait venir à soi. C'est que l'Ereignis cherche à dire le rapport entre l'Être et le Dasein, et cela, le titre du paragraphe 135 le dit bien: «Le déploiement de l'Être comme Ereignis (la relation du Dasein et de l'Être) ».

L'Ereignis est donc le rapport par excellence, l'accord qui les accorde tous deux l'un à l'autre, et ce faisant, les fait venir à soi, à ce qu'ils ont de propre, dans la mesure où jamais le Dasein n'est sans l'Être ni l'Être ne se déploie sans le Dasein, dans la mesure encore où « le Da-sein n’est que d'être le La de l'Être, le site de son déploiement », et où l'Être est caractérisé par la finitude qui signifie qu'il requiert l'homme, il le revendique comme Dasein et n'est rien d'autre que de s'ouvrir pour l'homme: «La relation du Da-sein à l'Être appartient au déploiement de l'Être lui-même, ce qui peut aussi se dire ainsi : l'Être requiert le Da-sein et ne se déploie pas sans cette venue à soi (Ereignung). » (F. Fédier, 2013, p. 292).

Cette relation n'est nullement la relation du sujet à un objet, le $D a$ sein n'étant pas le sujet de la philosophie moderne, car n'étant pas une sphère d'immanence, et l'Être n'étant pas un objet, mais étant l'inobjectivable par excellence qui rend possible toute objectivation d'un étant. Elle n'est pas la mise en relation de deux étants, qui pourraient être 
d'abord séparément et seraient ensuite réunis. Cette relation n'a donc pas lieu à un moment donné comme si elle pouvait ne plus avoir lieu. Les membres du rapport ici ne sont rien en dehors du rapport qu'est l'Ereignis. Pour faire entendre cette seconde signification, on a parfois proposé comme traduction " copropriation », « appropriement » ou « avènement ». Enfin, Heidegger fait remarquer dans Identité et différence qu'Ereignis renvoie au verbe eraügen dans lequel se laisse entendre das Auge, l'œil. Et en effet, l'Ereignis étant «cela » qui donne l'Être, il est cela qui ouvre à l'homme l'éclaircie, l'ouverture où de l'étant peut venir se manifester, donc se donner à voir.

À partir des « traités impubliés » et notamment des « Apports à la philosophie : De l'avenance», l'Ereignis devient le mot directeur de la pensée d'Heidegger, le nouveau nom du déploiement originel de l'Être. Alain Boutot dans son Que-sais-je ?, résume ainsi le sens de l'Ereignis :

"L'être comme le temps, entrent en présence ou plutôt, ne sont rien d'autre que la venue en présence de tout ce qui est. Cette présence ne dérive pas d'autre chose que d'elle-même. Elle advient d'elle-même et par elle-même, elle se donne, ou plutôt est elle-même pure donation de présence. Heidegger nomme cette donation originaire de la présence, qui est à la fois la vérité de l'être et la vérité du temps, das Ereignis » (A. Boutot, 1989, p. 58).

\section{L'Ereignis comme le mot-directeur de la pensée heideggérienne}

L'homme, selon Heidegger, est cet étant ontologiquement privilégié en ceci qu'il a toujours déjà une certaine entente de l'être, non une connaissance, mais une certaine compréhension implicite et non thématique de ce qui signifie "Être » pour les étants (les choses qui sont) qui l'entourent. La connaissance de l'étant est dite ontique : la science est un exemple de connaissance ontique en ce qu'elle n'interroge jamais les présupposés de ses relations aux objets. La question de l'être de l'étant, quant à elle, est dite ontologique.

«Être et temps », cet ouvrage essentiel de 1927 de Heidegger, interroge le sens de l'être, thème fondamental de l'ontologie, définie déjà par Aristote comme étant la question de l'être en tant qu'être. Pour Heidegger, celle-ci est tombée dans l'oubli et la trivialité (la tradition philosophique qu'il faudra détruire - ou, selon les traductions - déconstruire), et doit être reposée à la lumière d'une « analytique du Dasein » c'est- à- dire une étude structurelle de l'existence humaine.

L'analytique existentiale, qui consiste en l'examen des structures de l'être de cet étant appelé Dasein, se présente comme une voie d'accès au sens de l'être (M. Heidegger, 1986, § 2, p. 28). L'intention de Heidegger, dans « Être et temps », était dès le départ, de passer de l'élucidation de l'être 
de l'étant à celle de l'être en général. C'est la raison pour laquelle il a pris soin de définir sa recherche comme « une avancée en direction du concept d'être, et ce, par le chemin d'une interprétation spéciale d'un étant déterminé, le Dasein, à partir duquel doit être conquis l'horizon nécessaire à une compréhension possible de l'être » (M. Heidegger, 1986, § 8, p. 67).

En fait, tout se passe comme prévu. L'analytique existentiale s'achève sur la définition de l'être du Dasein comme totalité articulée et unifiée par le « souci ». Celui-ci apparaît comme une structure unifiante. Mais nous ne savons pas encore, à la fin de la première section, ce qui constitue ontologiquement l'unité de cette multiplicité. C'est la deuxième section qui se consacre à remonter du résultat de l'analytique existentiale à son fondement : « la question ontologique, écrit Heidegger au terme de son analyse du souci, doit être menée encore plus loin, jusqu'à la mise à découvert d'un phénomène encore plus original, qui porte ontologiquement l'unité et la totalité de la structure multiple du souci » (M. Heidegger, 1986, $\S 41$, p. 246). La question du sens de l'être se ramène donc à la question de savoir ce qui rend possible l'unité articulée par le souci.

En résumé, la première section a consisté à remonter de la diversité des modes d'être du Dasein à la totalité du souci, la deuxième s'est interrogée sur le fondement de l'unité de cette totalité. Au fil de l'analyse, il est apparu que les moments constitutifs du souci, être en avant de soi, être déjà, être auprès de, correspondent respectivement à l'avenir, au passé et au présent. Ces trois moments, avons-nous lu, ne s'ajoutent pas du dehors les uns aux autres mais s'emboîtent mutuellement selon un processus qui est la « temporalité ». Heidegger, pour mettre celle-ci en lumière, l'a soigneusement distinguée du temps ordinaire. La temporalité, en effet, ne doit pas être pensée à partir du temps, mais le temps à partir de la temporalité.

« Si l'on part, écrit Marlène Zarader, de la représentation traditionnelle du temps comme succession, on sera conduit à se représenter la temporalité sur le mode d'une accumulation progressive : de même que le maçon compose une maison avec des pierres, de même la temporalité composerait, avec les fragments du temps, le déroulement infini et monotone de ses moments. Et, telle la maison qui peut ensuite être démolie pierre par pierre, cette temporalité compacte serait susceptible d'être indéfiniment redivisée en chacune de ses parties » (M. Zarader, 1986, p. 212).

Le temps originaire ne saurait donc se confondre avec le temps ordinaire. En d'autres termes, la temporalité ne saurait être traitée comme un étant : « la temporalité n'est pas, elle se temporalise ». Elle est le processus temporel lui-même consistant à temporaliser ses modes. L'unification réalisée ne réside pas dans un rassemblement en soi de parties isolées, elle consiste à projeter hors de soi ses propres possibilités sans pour autant s'en 
séparer. Cette projection est l'effet de la temporalité qui est extériorisation, « l'en dehors de soi originel » (M. Heidegger, 1986, § 65, p. 391). Ainsi, la deuxième section a montré que le sens de l'être du Dasein, le souci, vient de la temporalité. Au terme de Sein und Zeit, nous avons donc été conduits du Dasein au Temps.

Le déroulement interne du traité montre qu'il conduisait au-delà. L'ambition de l'auteur était bien, dès le départ, de s'interroger sur l'être même. Nous avons compris, en effet, que l'objectif de l'analytique existentiale n'était pas de répondre à la question de l'être mais de la préparer. La deuxième section s'est achevée sur la même promesse. Nous sommes allés du Dasein au temps, mais pour arriver au but, il faut aller du temps à l'être. D'où l'ultime question sur laquelle se ferme le livre : « le temps se manifeste-t-il comme horizon de l'être ? ». Or, tel est le thème de la troisième section qui devait être intitulée «Zeit und Sein » et qui fera l'objet d'un séminaire paru sous ce titre. Nous restons donc en chemin. À quoi attribuer la cause de cette interruption ? Ni de ce que l'être y aurait été mal situé par rapport au temps, répond Zarader, ni de ce que le Dasein y aurait été mal situé par rapport à l'être. Mais ce qui se révèle impossible, ce fut de remonter de la temporalité comme être du Dasein à la mise à découvert du temps comme horizon de l'être (M. Heidegger, 1976, p. 216). La raison de cet « échec »se trouve dans le fait que la philosophie de Heidegger réclame un au-delà de l'être et du temps.

Pourquoi ce dépassement est-il exigé et comment se réalise-t-il ? L'être visé par Heidegger, nous l'avons lu, n'est pas un absolu séparé. Il est, à l'intérieur de sa propre histoire, l'ensemble des figures par lesquelles il se dispense. Dans le séminaire intitulé Zeit und Sein, Heidegger s'interroge sur la nature du rapport entre temps et être. Il ne peut s'agir d'une relation entre deux êtres, car du temps et de l'être on ne peut dire qu'ils sont. La seule chose que l'on puisse affirmer est qu'il y a, Es gibt, du temps et de l'être. Cette expression formée du verbe « Geben » qui signifie donner, devrait se traduire littéralement "cela donne ». Es gibt Sein veut donc dire ce qui donne l'être. Il y a donation d'être. «L'être se déploie comme l'histoire de sa propre dispensation, et cette dispensation - qui est l'être même - provient d'une donation préalable qui n'est plus du tout l'être et qui repose dans le Es » (M. Heidegger, 1976, p. 324).

Or, à partir du moment où l'être est donné ce qui donne ne peut être l'être ! Cet autre que l'être qui donne l'être c'est le temps, car le temps donne la présence qui se confond avec l'être. Mais si le temps donne l'être qui donnera le temps ? Ce ne peut être l'être puisque c'est le temps, puisque le temps ne se donne pas. Ce qui donne le temps et l'être, et les fait advenir, c'est le Es primordial. Mais le Es donateur d'être est autre que le Es donateur de temps. Et, cependant, il est aussi le même car ce qui donne le temps se 
révèle comme ce qui donne l'être. Le Es est à la fois le même et autre ! Il y a donc dans le $E s$ original comme une brisure, un écart, un « décalage intérieur à l'identité » (M. Heidegger, 1976, p. 242) qui explique le surgissement, l'avènement de l'être et du temps. Cet avènement, Heidegger l'appelle Ereignis.

L'Ereignis est ce qui fait s'entr'appartenir le temps et l'être. Il est le rapport de tous les rapports engendrés par cette co-appartenance. Celle-ci est rendue possible par la Différence installée au cœur du Même. Nous ne sommes donc pas dans une philosophie de l'Identité, où tout est pareil, égal, parce que rien ne diffère, et où tout est statique et vide pur. Nous sommes dans une philosophie du Même. Le Même suppose la différence de ce qui diffère et le rassemblement en lui de ce que la différence sépare. Alors que le pareil annule, par égalisation, les termes différents. Jean-Luc Marion peut écrire sans jeu de mots : « Le Même ne revient pas au pareil ni le pareil au Même » (J-L. Marion, 1983, p. 154).

Ni être, ni temps, l'Ereignis est le mouvement de « désappropriement » par lequel il se dépossède de soi au profit de ce qu'il donne. Ainsi, l'Ereignis est essentiellement retrait. Il se donne en se retirant. De lui, tout dérive et à lui tout retourne en s'abolissant. L'Ereignis apparaît comme l'origine de ce double mouvement de descente et de remontée qui va du temps à l'être, de l'être au Dasein, qui s'efface au profit du monde et des choses, puis des choses et du monde au Dasein, à l'être et de l'être au temps.

Ainsi, le temps et l'être s'effacent au profit de ce qui les dépasse : le Es gibt donateur originel. L'apparition évanouissante de l'être en faveur de l'Ereignis est le terme ultime de la pensée de Heidegger qui échoue, par conséquent, comme philosophe de l'être : « tandis que l'être vient au regard comme Ereignis, il disparaît en tant qu'être... L'Ereignis est bien plutôt pensé de telle sorte qu'il ne puisse être maintenu ni comme être ni comme temps » (J-L. Marion, 1983, p. 228). Heidegger n'est pas parvenu à penser l'être en tant qu'être. Lui-même en fit l'aveu à Jean Beaufret : " Dès la lettre sur l'humanisme, je continue à dire Sein, mais je pense Ereignis »(J-L. Marion, 1983, p. 229). Après « Être et temps », il y a eu un moment où Heidegger a profondément reconsidéré sa position sur plusieurs notions exposées dans « Être et temps ».

La philosophie de Heidegger, à la fois, renoue avec la grande tradition de l'antiquité en posant le problème de l'être en général et répond à la préoccupation de la pensée moderne de rendre à la personne la maitrise de son destin. Mais dans l'idéalisme occidental, la souveraineté du moi n'a jamais été séparable du prestige du Transcendant qu'il soit Dieu ou qu'il soit simplement l'Eternel mais toujours un Existant.

En posant le problème de l'ontologie où à juste titre Heidegger voit l'essentiel de son œuvre, il a subordonné la vérité ontique, celle qui se dirige 
sur l'autre, à la question ontologique qui se pose au sein du Même, de ce soimême qui, par son existence a une relation avec l'être qui est son être. Cette relation avec l'être est l'intériorité originelle véritable. La philosophie de Heidegger est donc une tentative de poser la personne en tant que lieu où s'accomplit la compréhension de l'être en renonçant à tout appui dans l'Eternel. Dans le temps originel, où dans l'être pour la mort, condition de tout être, elle découvre le néant sur lequel elle repose, ce qui signifie aussi qu'elle repose sur rien d'autre que sur soi. Royauté qui tient à notre indigence ; elle est sans triomphe et sans récompense.

Le débat de Heidegger avec la métaphysique peut souvent apparaître très complexe. Il est vrai que les textes de Heidegger sont parfois très obscurs. Mais son expérience fondamentale de l'être ressortit à quelque chose de très simple, voire à ce qu'il y a de plus élémentaire, à savoir qu'il est de l'être, de l'étant et non pas rien. Ce fut, aux yeux de Heidegger, la toute première expérience des penseurs grecs : il y a de l'être, l'être émerge et nous submerge. C'est la pensée, parménidienne aussi bien qu'héraclitéenne, de la phusis, mais c'est aussi une expérience qui est à la portée de chacun, s'il est vrai que le Dasein, que nous sommes tous, ne désigne rien d'autre que l'ouverture à l'être, mais toujours pour un temps, dont nous ne disposons pas.

Mais si cette merveille, pourtant élémentaire, n'est plus éprouvée comme telle, c'est, estime Heidegger, à cause de la pensée métaphysique, avide d'explication et de certitude. Face à l'être, toute explication arrive trop tard, elle n'arrive jamais à rattraper, encore moins à expliquer son émergence et à penser notre étonnement d'être là. L'essentiel du débat de Heidegger avec la métaphysique réside, peut-être, dans ce rappel, dans l'exploration d'un autre regard. Mais dans la mesure où il parvient ainsi à éveiller l'esprit à un mystère qu'il ne pourra jamais expliquer, il se pourrait qu'il reconduise aussi la métaphysique à sa plus haute possibilité, c'est-à-dire à l'admiration devant l'être. La philosophie n'a, peut-être pas de plus noble tâche. Heidegger répète la métaphysique tout en la dépassant.

Ces dernières décennies, l'observation d'un certain regain d'intérêt pour la philosophie témoigne de l'attente la plus légitime qui soit, eu égard aux prétentions traditionnelles et à l'aura de cette discipline, mais également la plus difficile à satisfaire : qu'elle mène celui qui épouse ses chemins à une véritable ré-appropriation de sa propre existence, à une re-création personnelle.

Impossible ici de se satisfaire d'un horizon de consolations aimables et de recettes de prospérité, non plus que de vertiges théorisant ou d'érudition monomaniaque. Face à un monde qui semble avoir d'avance consumé nos élans et consommé nos révoltes, les postures et commodités ne sont plus de mise. Un retour d'authenticité s'impose à l'engagement 
philosophique lui-même. En fait, la mise à l'épreuve de soi est passage obligé, dès lors que de mort de Dieu en chute des idoles de remplacement, nul ne peut plus prétendre faire surgir la valeur irréductible de l'humain d'ailleurs que de l'homme.

D'où la pertinence et l'attrait de l'exploration des voies traditionnelles et ressorts nouveaux d'une maïeutique propre à faire naître l'homme à lui-même, à travers la mise à nu et le retrait de tous les masques que nous imposent la vie en société et le respect de ses codes. Poussant au déracinement du moi comme à une étape nécessaire au dévoilement de notre vérité, nous nous inscrivons dans la lointaine filiation de ces philosophes de l'Antiquité (Stoïciens, Cyniques) qui vivaient leur philosophie comme un travail sur soi, voire une ascèse, et dans le prolongement de tout ce pan de la pensée contemporaine qui, avec Foucault notamment, s'est employé à armer la philosophie d'un pouvoir maximal de transformation croisée de celui qui s'y adonne et du social qui l'environne.

L'année du centenaire de la naissance de Heidegger en 1989, a été marquée par la publication, pour la première fois, chez l'éditeur Vittorio Klostermann, à Francfort, de l'un des ouvrages les plus puissamment révolutionnaires de toute l'œuvre de ce penseur majeur de notre temps: les Beiträge zur Philosophie (Vom Ereignis). Les Beiträge zur Philosophie, en français: les Apports, les Compléments ou les Contributions à la philosophie, sont un livre à part dans l'œuvre de Heidegger. Ils font partie des volumes écrits de 1936 à 1944 (parfois au-delà, jusque dans l'immédiat après-guerre), alors soigneusement recopiés, puis scellés et réservés, destinés à n'être éventuellement publiés que quelque cinquante ans après leur élaboration.

Les Beiträge sont même l'ouvrage de tête de toute la série de ces livres scellés, qui furent écrits au plus fort de l'une des plus sombres « époques » de l'histoire de l'Europe. La rédaction des Beiträge proprement dite s'étend de 1936 à 1937, avec adjonction d'un complément: "L'Estre » — «Das Seyn » — qui date de 1938. Cet ouvrage est essentiel parce qu'il touche à ce qui constitue le centre de gravité de tout le travail de Heidegger depuis le début des années 1930 jusqu'à l'extrême fin du chemin de pensée, renchérit Gérard Guest (2005). Il touche à la pensée de l'Ereignis — aux abords délicats de « la chose même » dont il s'y agit — et qui est de l'ordre de l'« Événement » (au sens très singulier que va prendre ce terme). Du reste, le livre porte en sous-titre, soigneusement placée entre parenthèses, la mention : «(Vom Ereignis)», ce que l'on pourrait tenter de traduire, en première approximation, par : « (De l'Événement)».

«Le mot « Ereignis » est, de l'aveu de Heidegger, le mot-clé de toute sa pensée depuis le début des années 1930 — celui autour duquel tourne toute la pensée du « tournant », de l'inflexion de la « Kehre »»(G. Guest, 
2005, p. 309). Concernant les ouvrages qui traitent ouvertement de 1'«Ereignis», tout semble s'être passé comme si Heidegger avait très longtemps préféré les maintenir soigneusement scellés, à distance du public, à commencer par celui de ses propres auditeurs et étudiants. Il semble avoir estimé qu'on ne pouvait alors, dans l'« état actuel des choses » (notamment : sous la chape de plomb du « national- socialisme »), leur faire accueil sans de très graves malentendus. La décision de réserver ainsi une part décisive de son travail de penser, est chez lui, quelque chose de mûrement médité.

S'il refuse d'envisager que ce qu'il écrit puisse même être publié avant très longtemps (et peut-être même envisage-t-il qu'il ne le soit jamais), ce n'est pas seulement pour des raisons qui tiendraient aux circonstances historiques, mais pour des raisons encore autrement essentielles (qui tiennent aux détours de l'« histoire de l'Estre »), et que l'effondrement même du « Troisième Reich » n'a manifestement pas suffi à rendre caduques. La nécessité - évidente — de soustraire la radicalité de sa méditation à l'impitoyable censure «nationale-socialiste » ne détermine qu'en partie pour Heidegger l'impossibilité de publier de son vivant quoi que ce soit de son ouvrage. Si le livre doit rester caché, dûment scellé, éventuellement mis à l'abri des bombardements - et très longtemps rendu inaccessible au public, c'est aussi et surtout eu égard à la nature même de l' "Événement » inouï que Heidegger ose y envisager, ainsi qu'aux abords délicats de « la chose même» dont il y est question, abords inaccessibles aux modes d'investigation qui demeurent marqués au coin de la métaphysique.

La prise en considération (vitale) du jeu (dangereux) avec les limites (très tranchantes) de la censure totalitaire n'explique pas à elle seule le recours au secret. C'est d'autant plus vrai que l'effondrement du nazisme, en 1945, ne donnera pas lieu à la publication (savamment retardée) de toute cette part décisive de son « grand œuvre », publication que le penseur souhaitera posthume. Sa conviction profonde, invoquée çà et là par Heidegger dans son livre même, est que la « pensée de l'Ereignis », à laquelle il se voit conduit à s'engager seul, ne saurait de longtemps être que profondément étrangère aux préoccupations de ses contemporains - qu'elle demeurera fatalement incomprise. Quand le monde entier raisonne dans les seuls termes de la «métaphysique de la volonté de puissance » portée à son comble, comment pourrait-il donc être question de procurer le moindre accès à ce dont il « s'agit » au cour de l'Ereignis! Cela paraît bien être manifestement impossible, ajoute Gérard Guest.

Pour se mettre en mesure de seulement envisager l' «Événement» de l'Ereignis qu'a désormais en vue Heidegger (et lui seul, à notre connaissance), il faudrait encore inventer, à même la langue commune (et à contre-courant de toute «langue savante» traditionnelle), une nouvelle modalité de la « langue » et de la « parole », capable de s'aventurer dans 
l'exploration de la structure mouvementée de l'« Événement » en question. C'est à cela — et à rien de moins — que se risque l'écriture des Beiträge. La structure mouvementée de l'Ereignis, selon Gérard Guest (2005, pp. 306372), la « singularité » absolue, jusqu'alors inaperçue, de « ce dont il s'y agit » comme à l'insu de tous, autant que l'impitoyable « dureté » inhérente à la période historique et aux ténèbres imminentes de l' « époque », imposent à Heidegger de mettre sous scellés, à l'intention d'un « futur » lointain, hypothétique et même plus qu'improbable, la part la plus risquée, la plus aventurée, de sa méditation — nécessairement solitaire.

Cette part scellée et réservée de l'œuvre de pensée de Heidegger comprend d'autres ouvrages, qui prolongent et accompagnent les Beiträge : Méditation du sens (Besinnung), écrit en 1938/1939, L'histoire de l'Estre (Die Geschichte des Seyns), de 1938 à 1940, ou bien encore Métaphysique et nihilisme (Metaphysik und nihilismus), écrit en 1938/1939, puis poursuivi entre 1946 et 1948. Ces trois ouvrages ont été publiés en allemand par l'éditeur Vittorio Klostermann, à la suite des Beiträge, à partir de 1989. D'autres encore suivront, ainsi Über den Anfang (1941), et Die Stege des Anfangs (1944), où il s'agit de méditer la frêle possibilité (la « Passerelle de l'Initial ») d'un « autre commencement de penser », lequel pût enfin espérer échapper à l'emprise du « premier commencement» (celui de « l'histoire de la métaphysique ») ; ou bien encore tout un volume intitulé Das Ereignis, de 1942, où il s'agit, bien entendu, de méditer la structure et les mouvements de l'« Événement » en question.

Il ne s'agit pas de Cours, ni non plus de recueils d'Essais et conférences (même si ces « écrits scellés », " impubliés », sont contemporains des grands Cours consacrés à Nietzsche et à Hölderlin, ainsi qu'à Parménide et à Héraclite). Non : il s'agit bien plutôt là de l'«œuvre» même — «das Werk» —, du «Livre» — «das Buch» — qui seul eût pu véritablement succéder au premier «livre» (inachevé) de Heidegger: Être et temps (1927). Il s'agit donc bel et bien là, après l'inachèvement manifeste d'Etre et temps (sans préjudice de quelque plus secret « achèvement latent »), de ce que Friedrich-Wilhelm von Herrmann a appelé « le second chefd'œuvre » de Heidegger. (Ce qui ne signifie nullement que ne doivent pas être comptés comme tels tous ces autres ouvrages dûment publiés.

Les Beiträge tiennent à l'intérieur de la part scellée de l'œuvre de Heidegger une place cruciale. Les autres volumes qui en sont parus à ce jour semblent ne devoir jamais faire qu'en expliciter les grandes intuitions et toutes les décisions majeures. Les Beiträge constituent le point où viennent se nouer tous les fils de la «méditation », " die Besinnung », dans laquelle s'engage désormais la pensée de l'Ereignis, pensée directrice de la philosophie de Heidegger, termine Gérard Guest (2005, pp. 306-372). 


\section{Conclusion}

Le déploiement de l'être n'est plus appréhendé à partir de la dimension compréhensive de notre être-le-là (Dasein) mais à partir de l'être lui-même, en son propre (Eigen). C'est pourquoi, avec les Beiträge, le terme Ereignis (événement-appropriement), à partir duquel l'être est pensé, devient le terme directeur de la pensée de Heidegger pour le rester jusqu'à la fin de son chemin de pensée (W.J. Richardson, 2011). Ce double tournant des années trente, celui provoqué par sa rencontre avec le dire poétique d'Hölderlin et celui des Beiträge dans lequel le langage est pensé à partir de l'être, ce dernier pensé à son tour à partir de l'Ereignis, est assurément décisif pour la question du langage. Et pourtant, il nous faut lire la note de bas de page insérée, trois ans plus tard, à sa lettre Über den Humanismus de 1946, dans le recueil Wegmarken, pour comprendre à quel point, même en 1946, le chemin est encore en chemin :

Das hier Gesagte ist nicht erst zur Zeit der Niederschrift ausgedacht, sondern beruht auf dem Gang eines Weges, der 1936 begonnen wurde, im $>$ Augenblick< eines Versuches, die Wahrheit des Seins einfach zu sagen Der Brief spricht immer noch in der Sprache der Metaphysik, und zwar wesentlich. Die andere Sprache bleibt im Hintergrund.

Ce qui est dit ici n'est pas seulement d'abord inventé au temps de sa mise par écrit, mais repose sur la marche d'un chemin, qui fut commencé en 1936, dans l'instant d'une tentative de simplement dire la vérité de l'être La lettre parle toujours et encore dans le langage de la métaphysique et, à la vérité, essentiellement. L'autre langage reste en arrière-fond. (M. Heidegger, 2013, p. 313).

Ainsi, le chemin de pensée de Heidegger, entamé dès ses réflexions de jeunesse en 1907 et qui connaît ce tournant majeur des années trente, continue pour le moins à se chercher et à rester insatisfait, car encore trop tributaire du langage métaphysique, en 1946. Cet autre langage, dont il est question, resté en arrière-fond, Heidegger ne se met en route vers son déploiement, et de manière "systématique » du moins, qu'au temps des séries de conférences tenues tout au long des années cinquante et recueillies sous l'intitulé : Unterwegs zur Sprache-Acheminement vers la parole.

Les propos tenus ci-dessus cherchent à montrer à la fois le suivi et l'évolution de la pensée de Heidegger, une pensée dont le chemin, en chemin, et restant sur le chemin, vient à se transformer, menant vers une éclaircie toujours plus claire. Heidegger se confie ainsi:

Ich folgte immer nur einer undeutlichen Wegspur, aber ich folgte. Die Spur war ein kaum vernehmbares Versprechen, das eine Befreiung ins Freie ankündete, bald dunkel und verwirrend, bald blitzartig wie ein jäher Einblick, der sich dann auf lange Zeit hinaus wieder jedem Versuch, ihn zu sagen, entzog. 
Je ne suivais toujours que la trace d'un chemin qui n'était pas distincte, mais je suivais. La trace était promesse à peine perceptible, annonce d'une libération dans le libre, tantôt obscure et embarrassante, tantôt soudain coup d'œil foudroyant, qui ensuite pour longtemps se dérobait à toute tentative de la dire (M. Heidegger, 1981, p. 137).

Ce que ce coup d'œil pénétrait soudainement de son regard, c'est le domaine du tout autre, là où l'être, envisagé en lui-même, débarrassé de l'entrave des conceptions métaphysiques, se déploie en sa liberté comme donation pure.

\section{References:}

1. Beaufret Jean (1985). Dialogue avec Heidegger: Tome 4, Le chemin de Heidegger, Paris, Editions de Minuit, 130 p.

2. Boutot Alain (1989). Heidegger, Paris, PUF, coll. « Que sais-je ?» (no 2480), $127 \mathrm{p}$.

3. Fedier François (2013). Entendre Heidegger et autres exercices d'écoute, Paris, Editions Pocket, 448 p.

4. Franck Didier (2004). Heidegger et le Christianisme : L'explication silencieuse, Paris, PUF, coll. « Epiméthée », 144 p.

5. Guest Gérard (2005). De l'événement même (De l'Ereignis) in Revue Ligne de risque, $\mathrm{N}^{\circ} 21$, collection « L'infini », Gallimard, Paris, pp. 306-372.

6. Heidegger Martin (1986). Etre et Temps, traduit de l'allemand au français par François Vézin, Tome I, Paris, Gallimard, 581 p.

7. Heidegger Martin (1981). Acheminement vers la parole, trad. François Fédier, coll. TEL, Paris, Gallimard, 260 p.

8. Heidegger Martin (2013). Apports à la philosophie : De l'avenance, trad. François Fédier, Paris, Gallimard, 624 p.

9. Heidegger Martin (1976). Questions IV, Trad. de Jean Beaufret, Paris, Gallimard, 343 p.

10. Heidegger Martin (1970). Lettre sur l'humanisme-Über den Humanismus, Trad. Roger Munier, Paris, Aubier éditions Montaigne, coll. « bilingue », $189 \mathrm{p}$.

11. Heidegger Martin (2013). Wegmarken, Edité par Verlag Vittorio Klostermann, Frankfurt am Main, 487 p.

12. Marion Jean-Luc (1983). « Du pareil au même " in Cahier de l'Herne, Paris, 1983, pp. 154 et 229.

13. Narbone Jean-Marc (2001). Hénologie, ontologie et Ereignis (Plotin Proclus Heidegger), Paris, Les Belles Lettres («L'âne d'or »), 384 p.

14. Pieron Julien (2010). "Immanence et transcendance dans la lecture heideggérienne d'Anaximandre " [archive], Klêsis - Revue philosophique, Montpellier, Université Paul Valery, pp.125-150. 
15. Pinat Etienne (2014). « Martin Heidegger: Apports à la philosophie (Partie I) » in Revue de Philosophie en ligne Actu Philosophia, mis en ligne le 22 janvier.

16. Richardson William John, S.J. (2011). Heidegger: Through Phenomenology to Thought, preface by Martin Heidegger (pp.8-23), Berlin, Springer Science \& Business Media, $768 \mathrm{p}$.

17. Varenne Jean (2017). " NĀGĀRJUNA (150 ?-? 250) ", Encyclopædia Universalis [en ligne], consulté le 2 mai. URL : http://www.univers a lis.fr/e ncyclope die/naga rjuna/

18. Zarader Marlène (1986). Heidegger et les paroles de l'origine, Paris, Vrin, $320 \mathrm{p}$. 